\title{
On accommodating the factors influencing the sports results by splitting the order effect in paired comparison experiments
}

\author{
Nasir Abbas \\ Department of Statistics, \\ Government Postgraduate College Jhang, Pakistan \\ nabbasgcj@yahoo.com \\ Muhammad Aslam \\ Department of Statistics, \\ Quaid-i-Azam University Islamabad, Pakistan \\ aslamsdqu@yahoo.com
}

\begin{abstract}
The results of sport contests depend upon a lot. In this article, an attempt is made to accommodate the factors influencing the sports-results by proposing a model for paired comparison experiments that splits the order effect into its components. The proposed model can be used to separately study the effects of all the components of the order effect. We study only two components of the order effect as a special case. The maximum likelihood estimates of the worth parameters are found and the plausibility of the proposed model is checked. Real dataset is collected on five top-ranked one-day-international cricket teams and is used to illustrate the estimation procedure.
\end{abstract}

Keywords: Bradley-Terry model; Goodness of fit; Home advantage; Maximum likelihood (ML) estimates; Paired comparisons; Ranking; Toss-result effect.

\section{Introduction}

In the method of paired comparisons (PC), treatments (stimuli, options, objects, items, individuals etc) are presented in pairs to judges (raters, respondents, jurists, panelists etc) that are asked to pick the better one on the basis of sensory evaluation. If allowed, they may declare a tie rendering the two objects equal in worth when the difference between their worth is less than a certain critical or threshold value. By repeating this experiment a fixed number of times on balanced or un-balanced pattern under similar conditions, the PC dataset is generated and is expressed as a preference matrix. This preference matrix is analyzed through the PC models. The PC models quantify the qualitative preferences in the form of worths or merits of treatments. It is primarily used for subjective judgments where quantitative measurement is impossible or impracticable. It's also used in many cases where there may be a substantial effect of sampling error on the measurements. Hence it is widely used by psychometricians. The most frequent application has been to sensory testing; especially taste testing, consumer tests, personal rating, and choice behavior etc.

The method of paired comparisons is also used often to rank different sport teams of different games like soccer, chess, cricket etc. Though in a broader sense, the home-ground effect may be viewed as an order effect in sensory evaluations studied by Davidson and Beaver (1977). David (1988) provides a 
detailed review of the literature pertaining to the topic. Kuk (1995) studies the home-ground effects. We propose a model which splits the so-called order effect into its components. Cricket is a famous game which is getting even more popularity day-by-day. There is a variety of factors affecting the game results. The most important being the home-ground and toss-results. As a particular case, we consider cricket and separately study home-ground and toss-results effects by following the criterion adopted by Kuk (1995). Recent developments made in this field comprise the construction of different paired comparison models and their extensions to include different factors affecting the preferences declared by respondents about the competing treatments. Thurstone (1927) assumes that the responses follow normal distribution but Bradley (1953) assumes the Logistic distribution to present their paired comparison models. Glenn and David (1960), Joe (1990), Joe (1991) and Henery (1992) consider the Thurstonian model to accommodate ties and home-ground (order) effects. Stern (1990a) and Stern (1990b) propose the gamma models for paired comparisons and apply them to sports datasets for the 1986 National League baseball season. Each game is treated as a comparison of two teams. Abbas and Aslam (2009) compare two Cauchy variables to develop the Cauchy PC model. Abbas and Aslam (2010) perform the Bayesian analysis of the gamma models for paired comparisons proposed by Stern (1990a). Rao-Kupper (1967) and DavidsonBeaver (1977) discuss the accommodation of ties and order effects in the Bradley-Terry model due to Bradley and Terry (1952). Huang et al. (2006) propose a generalized Bradley-Terry model to be used for competitions between two teams with an additional quality of combining the outcomes resulting through many competitions. Brozos-Vzquez et al. (2008) bring a novel approach to the theory of tournament rankings by combining two different theories that are widely used to establish rankings of populations after a given tournament. Glickman (2008) develops an adaptive approach for pairing players in each round, in which the probability that the best player advances to the next round, is maximized. Abbas and Aslam (2011) accommodate quantitative weights in the Bradley-Terry model and declare it as a reconciliation between the qualitative and qualitative paired comparisons.

Current study may be outlined as follows: Section 2 deals with the construction of the proposed model. Section 3 estimates the model parameters using the classical ML approach. An illustrative example of estimation procedure is presented in Section 4. Section 5 checks the plausibility of the proposed model. The findings are discussed along with conclusions in the last Section 6.

\section{The model}

In this study, we propose a PC model by considering the toss-result (i.e. winning or losing a toss) effects in addition to the home-ground effects in cricket. The proposed model is an extension of the Kuk (1995) model. We ignore tie and accommodate/split the order effect into its contributing factors. By studying these factors, a clearer picture of the worth and ranking order of the objects under study may be seen. The study of accommodating the effect of all possible factors in paired comparisons is analogous to the study of the multiple-regression, where 
all possible important factors (repressors) are included and studied for their possible effect on the dependent variable.

The game of cricket is a popular games of the world and is getting even more popularity and fame. The answer to the question that "Which team will win" depends on a variety of factors; the most important being the grounds at which the matches are played and the toss-results. The ground-effect, which is fully recognized and admitted, may be classified as the home-ground and awayground effects, and has been studied by many authors. It may be declared parallel or equivalent to the order effect. We consider the toss-result as an additional important factor affecting the match results.

In addition to the home-ground effects, there is a significant effect of toss-results. Some important reasons for considering the effects of toss-results include: (i) the nature of pitch, where it become clear to the game-experts that which team may get benefits over the opponent by batting first or chasing. Since availing this benefit by opening first or chasing purely depends upon the toss-results; hence toss-results are important for getting these benefits. (ii) The playing-hours of a match (day match or day-night match) is another factor to consider the tossresults. There are no two opinions that the day-light is more preferable and favorable to the artificial lights. The natural day-light is ideal and perfect to watch the movement of the ball and is much more preferable as compared to the artificial lights. Hence every team wants to get the benefit of day-light by playing first in the day-and-night matches. Clearly, availing of this benefit solely depends upon the toss-results. (iii) Extreme weather is another factor. Every team wants to avoid the adverse effects of weather. In cold atmosphere, pitch and ground get wet and humid in the early day-time causing a hindrance in the movement of the ball, but this effect diminishes by sun-light with the passage of time. So, in order to avoid such adverse effects, the toss-winning team accordingly decides to bat first or field first. (iv) Batting and fielding are tasks of different natures causing varying degrees of tiredness and exertion. Both of the teams make a prior decision about batting first or chasing the opponent conditional upon the tossresults. Some teams have got more expertise either in batting first or chasing the opponent. They may also utilize this expertise by their will subject to the tossresults. There might be certain other effects of the toss-results, which the gameexperts are aware of. Hence it becomes quite evident from the above discussion that the toss-results are substantially important for the competing teams towards shaping the destination of their victories. Hence it becomes necessary to incorporate the toss-result effects in the study of the paired comparisons. In addition to the ground effects, an attempt is made in this article to consider the toss-result effects in the light of above motivating factors.

Prior to discussing the proposed model, the notations used therein are explained here. Let $F$ be the vector of factors indicating the presence of some attributes of interest and $F^{\prime}$ stands for its complement (absence of the attributes). Following the criterion of using the Logistic distribution for building the Bradley-Terry model, the proposed model takes the form: 


$$
\phi_{i j}^{F}=H\left(V_{i}^{F}-V_{j}^{F^{\prime}}\right)=\frac{1}{4} \int_{-\left(V_{i}^{F^{F}}-V_{j}^{F^{\prime}}\right)}^{\infty} \sec h^{2}\left(\frac{1}{2} x\right) d x=\frac{\theta_{i}^{F}}{\theta_{i}^{F}+\theta_{j}^{F^{\prime}}},
$$

for all $i(\neq j)=1, \ldots, t$. Here $t$ denotes the total number of treatments to be compared, $V_{i}^{F}=\log \left(\theta_{i}^{F}\right), \theta_{i}^{F}>0$ and denotes the worth or merit of treatment $i$ in the presence of a set of $p$ attributes, i.e., $F=\left(f_{1}, f_{2}, \ldots, f_{p}\right)$ for $i=1, \ldots, t, \phi_{i j}^{F}$ represents the probability of preferring treatment $i$ over $j$ in the presence of the set of attributes $F$ and its complementary probability is $\phi_{j i}^{F^{\prime}}=1-\phi_{i j}^{F}$. We further assume that all the attributes are uncorrelated with each other. Model (1) may be used to study the effect of the vector of $F$ attributes separately.

Being specific for the game of cricket, we take $p=2$ and consider only two attributes, i.e., the (home and away from home) ground effect, $H$, and the tossresult effect, $T$. The possible combinations comprise $H T, H L, G T$ and $G L$ respectively referring to the situations when a team is host and has won the toss, host and has lost the toss, guest and has won the toss and guest and has lost the toss, and $\boldsymbol{\theta}=\left(\theta_{i}^{H T}, \theta_{i}^{G L}, \theta_{i}^{H L}, \theta_{i}^{G T}\right)$ stands for the vector of the team-worths in the respective conditions. The specific form of the model (1) is:

$$
\phi_{i j}^{H T}=H\left(V_{i}^{H T}-V_{j}^{G L}\right)=\frac{1}{4} \int_{-\left(V_{i}^{H T}-V_{j}^{G L}\right)}^{\infty} \sec h^{2}\left(\frac{1}{2} x\right) d x=\frac{\theta_{i}^{H T}}{\theta_{i}^{H T}+\theta_{j}^{G L}},
$$

for all $i(\neq j)=1, \ldots, t$. Like before, here $t$ denotes the total number of treatments to be compared, $V_{i}^{H T}=\log \left(\theta_{i}^{H T}\right)$. Similarly $\phi_{i j}^{H L}=\frac{\theta_{i}^{H L}}{\theta_{i}^{H L}+\theta_{j}^{G T}}, \phi_{i j}^{G T}=\frac{\theta_{i}^{G T}}{\theta_{i}^{G T}+\theta_{j}^{H L}}$, and $\phi_{i j}^{G L}=\frac{\theta_{i}^{G L}}{\theta_{i}^{G L}+\theta_{j}^{H T}}$. The toss and home-ground effects for different teams may be viewed as the difference between their worth under relative conditions. For example, toss-effect may be the difference $\theta_{i}^{H T}-\theta_{i}^{H L}$ and home-ground effect may be visualized via $\theta_{i}^{H T}-\theta_{i}^{G T}$ and the joint home-toss effect through $\theta_{i}^{H T}-\theta_{i}^{G L}$.

The Thurstonian version of the paired comparison model takes the form $\phi_{i j}^{F}=\Phi\left(\theta_{i}^{F}-\theta_{j}^{F^{\prime}}\right)$, where $\Phi$ mirrors the cumulative probability of the normal distribution, and rest of the notations are as defined earlier. The Kuk's model under the split order-effect may be expressed as:

$$
\left.\begin{array}{l}
\phi_{i j}^{H T}=\Phi\left(\theta_{i}^{H T}-\theta_{j}^{G L}\right), \phi_{j i}^{G L}=\Phi\left(\theta_{j}^{H L}-\theta_{i}^{G T}\right) \\
\phi_{i j}^{H L}=\Phi\left(\theta_{i}^{H L}-\theta_{j}^{G T}\right), \phi_{j i}^{G T}=\Phi\left(\theta_{j}^{G T}-\theta_{i}^{H L}\right)
\end{array}\right\},
$$

where all the notations posses their usual definitions. 


\section{Classical ML estimation}

The classical ML estimates of the model parameters are found. The likelihood function for the proposed model under the above-defined probabilities takes the form

$$
L(\mathbf{a}, \quad)=K_{i j} \prod_{1 \leq i<j \leq t}\left(\phi_{i j}^{H T}\right)^{a_{j j}^{G T}}\left(\phi_{j i}^{G L}\right)^{a_{i j}^{G L}}\left(\phi_{i j}^{H L}\right)^{a_{i j}^{H L}}\left(\phi_{j i}^{G T}\right)^{G_{j i}^{G T}}\left(\phi_{j i}^{H T}\right)^{a_{j i}^{H T}}\left(\phi_{i j}^{G T}\right)^{a_{i j}^{G T}}\left(\phi_{j i}^{H L}\right)^{a_{i j}^{H L}}\left(\phi_{i j}^{G L}\right)^{a_{j j}^{G L}},
$$

where $a_{i j}^{H T}$ stands for the number of matches won by the host and toss-winner team $i$ against $j, a_{i j}^{G L}$ represents the number of matches won by the guest and toss-loser team $i$ against $j, a_{i j}^{H L}$, shows the number of matches won by the host and toss-loser team $i$ against $j$ and $a_{i j}^{G T}$ indicates the number of matches won by the guest and toss-winner team $i$ against $j$. Generally speaking, in the notations $a_{i j}^{H T}, a_{i j}^{G L}, a_{i j}^{H L}, a_{i j}^{G T}$, the superscripts are related to the first letter in the subscript and indicate the winner. Moreover, $n_{i j}^{H T}, n_{i j}^{G L}, n_{i j}^{H L}$ and $n_{i j}^{G T}$ denote the total number of matches played between teams $i$ and $j$ under the respective situations. $K_{i j}=\prod_{1 \leq i<j \leq t}\left\{\left(\begin{array}{c}n_{i j}^{H T} \\ a_{i j}^{H T}\end{array}\right)\left(\begin{array}{c}n_{i j}^{H L} \\ a_{i j}^{H L}\end{array}\right)\left(\begin{array}{c}n_{j i}^{H T} \\ a_{j i}^{H T}\end{array}\right)\left(\begin{array}{c}n_{j i}^{H L} \\ a_{i j}^{H L}\end{array}\right)\right\} \quad$ is the normalizing constant and is independent of the model parameters.

Expression (4) may be written as

$$
\begin{aligned}
L(\mathbf{a}, \mathbf{)})= & \prod_{1 \leq i<j \leq t} K_{i j}\left(\frac{\theta_{i}^{H T}}{\theta_{i}^{H T}+\theta_{j}^{G L}}\right)^{a_{i j}^{H T}}\left(\frac{\theta_{j}^{G L}}{\theta_{i}^{H T}+\theta_{j}^{G L}}\right)^{a_{j i}^{G L}}\left(\frac{\theta_{i}^{H L}}{\theta_{i}^{H L}+\theta_{j}^{G T}}\right)^{a_{i j}^{H L}}\left(\frac{\theta_{j}^{G T}}{\theta_{i}^{H L}+\theta_{j}^{G T}}\right)^{a_{j i}^{G T}} \times \\
& \left(\frac{\theta_{j}^{H T}}{\theta_{j}^{H T}+\theta_{i}^{G L}}\right)^{a_{j i}^{H T}}\left(\frac{\theta_{i}^{G L}}{\theta_{i}^{G L}+\theta_{j}^{H T}}\right)^{a_{i j}}\left(\frac{\theta_{j}^{H L}}{\theta_{j}^{H L}+\theta_{i}^{G T}}\right)^{a_{j i}^{H L}}\left(\frac{\theta_{i}^{G T}}{\theta_{j}^{H L}+\theta_{i}^{G T}}\right)^{a_{i j}},
\end{aligned}
$$

which simplifies further to:

$$
\begin{aligned}
L(\mathbf{a}, \quad) & =K_{i j} \prod_{i=1}^{t}\left\{\left(\theta_{i}^{H T}\right)^{a_{i}^{H T}}\left(\theta_{i}^{H L}\right)^{a_{i}^{H L}}\left(\theta_{i}^{G T}\right)^{a_{i}^{G T}}\left(\theta_{i}^{G L}\right)^{a_{i}^{G L}}\right\} \times \\
& \left\{\prod_{i(<)=1}^{t}\left(\theta_{i}^{H T}+\theta_{j}^{G L}\right)^{n_{i j}^{H T}}\left(\theta_{i}^{H L}+\theta_{j}^{G T}\right)^{n_{i j}^{H L}}\left(\theta_{i}^{G T}+\theta_{j}^{H L}\right)^{n_{i j}^{G T}}\left(\theta_{i}^{G L}+\theta_{j}^{H T}\right)^{n_{i j}^{G T}}\right\}^{-1},
\end{aligned}
$$

where $\quad n_{i j}^{H T}=a_{i j}^{H T}+a_{j i}^{G L}, \quad n_{i j}^{H L}=a_{i j}^{H L}+a_{j i}^{G T}, \quad n_{i j}^{G T}=a_{i j}^{G T}+a_{j i}^{H L}, \quad n_{i j}^{G L}=a_{i j}^{G L}+a_{j i}^{H L}$, $a_{i}^{H T}=\sum_{j(<i)=1}^{t} a_{i j}^{H T}+\sum_{j(>i)=1}^{t} a_{j i}^{G L}$, $a_{i}^{G T}=\sum_{j(>i)=1}^{t} a_{i j}^{G T}+\sum_{j(<i)=1}^{t} a_{j i}^{H L}, \quad a_{i}^{G L}=\sum_{j(>i)=1}^{t} a_{i j}^{G L}+\sum_{j(<i)=1}^{t} a_{j i}^{H T}$, $\boldsymbol{\theta}=\left(\theta_{i}^{H T}, \theta_{i}^{G L}, \theta_{i}^{H L}, \theta_{i}^{G T}\right)$ for all $i=1,2, \ldots, t$. The logarithm of the likelihood function is: 


$$
\begin{aligned}
\ln L(\mathbf{a}, \quad)= & \ln K_{i j}+\sum_{i=1}^{t} a_{i}^{H T} \ln \left(\theta_{i}^{H T}\right)+\sum_{i=1}^{t} a_{i}^{H L} \ln \left(\theta_{i}^{H L}\right)+\sum_{i=1}^{t} a_{i}^{G T} \ln \left(\theta_{i}^{G T}\right)+\sum_{i=1}^{t} a_{i}^{G L} \ln \left(\theta_{i}^{G L}\right) \\
& \quad-n_{i j}^{H T} \sum_{i(<)=1}^{t} \ln \left(\theta_{i}^{H T}+\theta_{j}^{G L}\right)-n_{i j}^{H L} \sum_{i(<)=1}^{t} \ln \left(\theta_{i}^{H L}+\theta_{j}^{G T}\right)-n_{i j}^{G T} \sum_{i(<)=1}^{t} \ln \left(\theta_{i}^{G T}+\theta_{j}^{H L}\right) \\
- & n_{i j}^{G T} \sum_{i(<)=1}^{t} \ln \left(\theta_{i}^{G L}+\theta_{j}^{H T}\right) .
\end{aligned}
$$

For the ML estimates, we maximize (6) with regards to the vector of unknown parameters $\boldsymbol{\theta}=\left(\theta_{i}^{H T}, \theta_{i}^{G L}, \theta_{i}^{H L}, \theta_{i}^{G T}\right)$. So we equate to zero the first partial derivatives of (6) with respect to the vector of unknown parameters $\boldsymbol{\theta}$. The set of likelihood equations, thus obtained, is:

$$
\left.\begin{array}{l}
\frac{a_{i}^{H T}}{\hat{\theta}_{i}^{H T}}-\sum_{i<j}\left(\frac{n_{i j}^{H T}}{\hat{\theta}_{i}^{H T}+\hat{\theta}_{j}^{G L}}\right)=0, \frac{a_{i}^{G L}}{\hat{\theta}_{i}^{G L}}-\sum_{i<j}\left(\frac{n_{i j}^{G L}}{\hat{\theta}_{i}^{G L}+\hat{\theta}_{j}^{H T}}\right)=0 \\
\frac{a_{i}^{H L}}{\hat{\theta}_{i}^{H L}}-\sum_{i<j}\left(\frac{n_{i j}^{H L}}{\hat{\theta}_{i}^{H L}+\hat{\theta}_{j}^{G T}}\right)=0, \frac{a_{i}^{G T}}{\hat{\theta}_{i}^{G T}}-\sum_{i<j}\left(\frac{n_{i j}^{G T}}{\hat{\theta}_{i}^{G T}+\hat{\theta}_{j}^{H L}}\right)=0
\end{array}\right\},
$$

which after simplification yield:

$$
\left.\begin{array}{l}
\hat{\theta}_{i}^{H T}=a_{i}^{H T} / \sum_{i<j}\left(\frac{a_{i}^{H T}}{\hat{\theta}_{i}^{H T}+\hat{\theta}_{j}^{G L}}\right), \hat{\theta}_{i}^{G L}=a_{i}^{G L} / \sum_{i<j}\left(\frac{a_{i}^{G L}}{\hat{\theta}_{i}^{G L}+\hat{\theta}_{j}^{H T}}\right) \\
\hat{\theta}_{i}^{H L}=a_{i}^{H L} / \sum_{i>j}\left(\frac{a_{i}^{H L}}{\hat{\theta}_{i}^{H L}+\hat{\theta}_{j}^{G T}}\right), \hat{\theta}_{i}^{G T}=a_{i}^{G T} / \sum_{i>j}\left(\frac{a_{i}^{G T}}{\hat{\theta}_{i}^{G T}+\hat{\theta}_{j}^{H L}}\right)
\end{array}\right\} .
$$

We use the restriction $\sum_{i=1}^{t} \theta_{i}^{H T}=\sum_{i=1}^{t} \theta_{i}^{G L}=\sum_{i=1}^{t} \theta_{i}^{H L}=\sum_{i=1}^{t} \theta_{i}^{G T}=1$ to avoid intractability.

Solution to the set of equations ( 7$)$ is obtained iteratively as follows.

If $\hat{\theta}_{i}^{H T(k)}$ is the $k$ th approximation to $\theta_{i}$, then

$$
\hat{\theta}_{i}^{H T(k)}=\hat{\theta}_{i}^{* H T(k)} / \sum_{i=1}^{t} \hat{\theta}_{i}^{* H T(k)} \text {, where } \theta_{i}^{* H T(k)}=a_{i}^{H T} / \sum_{i(<j)=1}^{t}\left(\frac{n_{i j}^{H T}}{\hat{\theta}_{i}^{* H T(k-1)}+\hat{\theta}_{j}^{* G L(k-1)}}\right) \text {. }
$$

The approximations for the other three categories may similarly be obtained. Following Bradley (1984), the iteration is started with initial specification of $\hat{\theta}_{i}^{H T(0)}=\hat{\theta}_{i}^{H L(0)}=\hat{\theta}_{i}^{G T(0)}=\hat{\theta}_{i}^{G L(0)}=1 / t$, for all $i=1,2, \ldots, t$.

\section{Illustrative example}

For illustration of the entire estimation procedure, we let $t=5$ and use a real dataset on five one-day-international (ODI) cricket teams of Australia (AU), India (IN), New Zealand (NZ), Pakistan (PA) and South Africa (SA) for the years 20002009. The dataset is collected through the website www.howstat.com. The 
subscripts $i, j=1,2, . ., 5$ respectively denote the teams of Australia, India, New Zealand, Pakistan and South Africa.

Table 1: Dataset of ODI matches with home-ground and toss effects

\begin{tabular}{lrrrrrrrr}
\hline Team Pairs & $a_{i j}^{H T}$ & $a_{j i}^{G L}$ & $a_{i j}^{H L}$ & $a_{j i}^{G T}$ & $a_{j i}^{H T}$ & $a_{i j}^{G T}$ & $a_{j i}^{H L}$ & $a_{i j}^{G L}$ \\
\hline (AU, IN) & 6 & 2 & 5 & 2 & 2 & 6 & 2 & 6 \\
(AU, NZ) & 3 & 2 & 7 & 4 & 3 & 6 & 2 & 5 \\
(AU, PA) & 8 & 2 & 2 & 2 & 0 & 0 & 0 & 0 \\
(AU, SA) & 4 & 4 & 4 & 2 & 4 & 3 & 5 & 6 \\
(IN, NZ) & 1 & 1 & 0 & 0 & 3 & 3 & 3 & 2 \\
(IN, PA) & 2 & 5 & 3 & 2 & 3 & 5 & 1 & 3 \\
(IN, SA) & 2 & 1 & 3 & 3 & 6 & 0 & 0 & 1 \\
(NZ, PA) & 5 & 1 & 2 & 2 & 5 & 0 & 3 & 0 \\
(NZ, SA) & 1 & 0 & 4 & 1 & 7 & 2 & 4 & 0 \\
(PA, SA) & 2 & 4 & 2 & 2 & 3 & 0 & 4 & 2 \\
\hline
\end{tabular}

Here $\theta_{i}^{H T}, \theta_{i}^{H L}, \theta_{i}^{G T}$ and $\theta_{i}^{G L}$ respectively denote a team-worth at home and tosswin status, home and toss-lost status, away-from-home and toss-won status and away-from-home and toss-lost status of the ith team.

We solve the set of equations (7) iteratively with initial value of $1 / t$ for all categories of the parameters $\theta_{i}^{H T}, \theta_{i}^{H L}, \theta_{i}^{G T}$ and $\theta_{i}^{G L}$, for $i=1,2, \ldots 5$. For identification, we impose the restrictions $\sum_{i=1}^{t} \theta_{i}^{H T}=\sum_{i=1}^{t} \theta_{i}^{H L}=\sum_{i=1}^{t} \theta_{i}^{G T}=$ $\sum_{i=1}^{t} \theta_{i}^{G L}=1$. We run a computer program developed in $C$ language $^{1}$ and the results thus obtained are displayed in Table 2.

Table 2: Parameter estimates

\begin{tabular}{lcccccccc}
\hline Teams & $\theta_{i}^{H T}$ & $\theta_{i}^{G T}$ & $\theta_{i}^{H L}$ & $\theta_{i}^{G L}$ & $\begin{array}{c}\text { Mean } \\
\text { Worth }\end{array}$ & $\begin{array}{c}\text { Home } \\
\text { Effect }\end{array}$ & $\begin{array}{c}\text { Toss } \\
\text { Effect }\end{array}$ & $\begin{array}{c}\text { Home-Toss } \\
\text { Effect }\end{array}$ \\
\hline Australia & 0.3004 & 0.2349 & 0.3359 & 0.2820 & 0.2883 & 0.0597 & -0.0413 & 0.0184 \\
India & 0.1477 & 0.1712 & 0.1124 & 0.1495 & 0.1452 & -0.0303 & 0.0285 & -0.0018 \\
New Zealand & 0.1238 & 0.1815 & 0.1204 & 0.2169 & 0.1606 & -0.0771 & -0.0160 & -0.0930 \\
Pakistan & 0.2454 & 0.1611 & 0.2173 & 0.0716 & 0.1739 & 0.1150 & 0.0588 & 0.1738 \\
South Africa & 0.1827 & 0.2513 & 0.2140 & 0.2801 & 0.2320 & -0.0674 & -0.0301 & -0.0974 \\
\hline
\end{tabular}

From the estimates, it reveals that Australia, on the average, is number one; South Africa is the second; Pakistan, the third; New Zealand, the fourth and India being the fifth and last one. Furthermore, different teams differently enjoy of or suffer from the ground and toss-result effects. The positive and negative values

\footnotetext{
${ }^{1}$ The program is not given here to reduce bulk, but may be had from the authors on request
} 
indicate positive and negative effects respectively. It is further interesting to note that the home-advantage after winning a toss is equivalent to home-advantage after losing the toss. Similarly, the toss-winning advantage at home is equivalent to the toss winning advantage at foreign grounds. Moreover, it is also seen that home-ground and/or toss-won penalize all the teams excepting Pakistan and Australia that have got the maximum benefit of their home-grounds. Pakistan has availed the toss-results to the maximum. Pakistan remains the highest in collectively availing the ground and toss-winning effects. It is worth mentioning that intrinsically the home-ground effects and the toss-result effects must be positive for the teams availing these effects. However, we have observed negative signs for these effects for some of the teams under consideration. Such a contradiction may be due to the reasons that there are numerous momentous factors, like the unfitness of an important player, misunderstanding between the two players while running on the pitch for getting a run and much more, which outweigh the home-ground and toss-result effects. The negative values may be attributed to such potential reasons.

\section{Test of adequacy of the model}

The adequacy of a model depends upon the closeness of the observed and the corresponding expected frequencies. The closer the observed and expected frequencies are, the smaller be the value of $\chi^{2}$, and the more adequate the model would be. Let the ordered pairs $\left(a_{i j}^{H T}, \hat{a}_{i j}^{H T}\right),\left(a_{i j}^{H L}, \hat{a}_{i j}^{H L}\right),\left(a_{i j}^{G T}, \hat{a}_{i j}^{G T}\right)$ and $\left(a_{i j}^{G L}, \hat{a}_{i j}^{G L}\right)$ denote the observed and the corresponding expected frequencies of preferences of the treatments $i$ over $j$ for under all categories. The $\chi^{2}$-statistic attains the form:

$$
\chi^{2}=\sum_{i \neq j}^{t}\left\{\frac{\left(a_{i j}^{H T}-\hat{a}_{i j}^{H T}\right)^{2}}{\hat{a}_{i j}^{H T}}+\frac{\left(a_{i j}^{H L}-\hat{a}_{i j}^{H L}\right)^{2}}{\hat{a}_{i j}^{H L}}+\frac{\left(a_{i j}^{G T}-\hat{a}_{i j}^{G T}\right)^{2}}{\hat{a}_{i j}^{G T}}+\frac{\left(a_{i j}^{G L}-\hat{a}_{i j}^{G L}\right)^{2}}{\hat{a}_{i j}^{G L}}\right\}
$$

with $4\{(t-1)(t-2) / 2\}=2(t-1)(t-2)$ degrees of freedom, (Stern (1990a) and Abbas and Aslam (2011)). The degrees of freedom are added due to the reproductive property of the chi-square distribution, Johnson et al. (1995)Error! Reference source not found.. The expected frequencies may be found as $\hat{a}_{i j}^{H T}=n_{i j}^{H T} \frac{\theta_{i}^{H T}}{\theta_{i}^{H T}+\theta_{j}^{G L}}$ and $\hat{a}_{j i}^{G L}=n_{i j}^{H T}-a_{i j}^{H T}$. The rest of the expected frequencies

may similarly be found. Through usual calculations, we find a $\chi^{2}$ value of 25.499796 with a highly insignificant $p$-value of 0.96374 . Hence we conclude that under no circumstances, the proposed model may be regarded as inadequate.

\section{Discussion and Conclusion}

A model for paired comparisons is proposed to split the order effect appearing in the paired comparison experiments. Actually, it is a Generalization/extension of the Kuk (1995) model ignoring ties; however we use logistic approach instead of the probabilistic one. The classical ML estimates of the model parameters are 
found using the iterative procedure and plausibility of the proposed model is also checked. It is also seen that if we average out the effects of grounds and tossresults affecting the performance, we get the estimates of worth equivalent to those produced by the renowned Bradley-Terry model. Davidson and Beaver (1977) study the effect of the order of presentation by considering it constant for all the treatments/teams, but our model has the potential of separately examining this effect for all possible pairs. Its significance in sports-science lies in the fact that it provides a better understanding of the worth of the competing treatments under different situations and helps understand the worth of the treatments under different scenarios. The results produced by the analysis may help the teams in paying more attention to cope with the odd situations generated at the playgrounds and make necessary improvements in their deficiencies. Moreover, a high $p$-value yielded in testing the adequacy of the proposed model also justifies the proposed extension in the model.

\section{Acknowledgement}

The authors would like to thank the reviewers for their potential comments on the article that lead to a significant improvement therein.

\section{References}

1. Abbas, N. and Aslam, M. (2009). Prioritizing the Treatments through Paired Comparison Models, A Bayesian Approach, Pakistan Journal of Statistics 25(1), 59-69.

2. Abbas, N. and Aslam, M. (2010). Bayesian Analysis of Gamma Models for Paired Comparisons, Pakistan Journal of Statistics, 26(3), 479-491.

3. Abbas, N. and Aslam, M. (2011). Extending the Bradley-Terry Model for Paired Comparisons to Accommodate Weights. Journal of Applied Statistics, 38(3), 571-580.

4. Bradley, R.A. and Terry, M.E. (1952). Rank Analysis of Incomplete Block designs: I. the Method of Paired Comparisons, Biometrika 39, 324-345.

5. Bradley, R.A. (1953). Some statistical methods in taste testing and quality evaluation, Biometrics 9, 22-38.

6. Bradley, R.A. (1984). Paired comparisons: some basic procedures and examples, In: Krishnaiah, P.R., Sen, P.K. (Eds.), Handbook of Statistics, Vol. 4. Amsterdam, North-Holland, 299-326.

7. Brozos-Vzquez, M., Campo-Cabana, M. A., Daz-Ramos, J. C. and Gonzlez-Daz, J. (2008). Ranking participants in tournaments by means of rating functions. Journal of Mathematical Economics, 44, 1246-1256.

8. David, H.A. (1988). The Method of Paired Comparisons, 2nd ed, Charles Griffin \& Company Ltd, London.

9. Davidson, R.R. and Beaver, R.J. (1977). On extending the Bradley-Terry model to incorporate within-pair order effects, Biometrics 33, 693-702. 
10. Glenn, W.A. and David, H.A. (1960). Ties in Paired Comparison experiments using a modified Thurstone-Mosteller Model. Biometrics, 16, 86-109.

11. Glickman, M. E. (2008). Bayesian locally optimal design of knockout tournaments. Journal of Statistical Planning and Inference, 138, 2117-2127.

12. Henery, R, J. (1992). Extension of Thurstone-Mosteller model for chess. The Statistician, 41, 559-567.

13. Huang, T., Weng, R. C. and Lin, C. (2006). Generalized Bradley-Terry Models and Multi-Class Probability Estimates. Journal of Machine Learning Research, 7, 85-115.

14. Joe, H. (1990). Extended use of paired comparison Models, with application to chess ranking. Applied Statistics, 39, 1, 85-93.

15. Joe, H. (1991). Rating systems based on paired comparison models. Statistics and Probability Letters, 11(4), 343-347

16. Johnson, N.L. Kotz S. and Balakrishnan, N. (1995). Continuous univariate distributions, Vol.2, $2^{\text {nd }}$ ed, John Wiley \& Sons, Inc.

17. Kuk, A.Y.C. (1995). Modeling Paired comparisons comparison data with large number of draws and Large Variability of Draw Percentages among Players. The Statistician, 44(4), 523-528.

18. Rao, P.V. and Kupper, L.L. (1967). Ties in paired-comparison experiments: a generalization of the Bradley-Terry model. Journal of American Statistical Association, 62, 194-204.

19. Stern, H. (1990a). A continuum of paired comparison models, Biometrika, $77,265-273$.

20. Stern, H. (1990b). Models for Distribution on Permutations. Journal of American Statistical Association, 85, 558-564.

21. Thurstone, L.L. (1927). A law of comparative judgment. Psychology Review, 34, 273-286. 